\title{
Biotechnological

\section{On the prevalance of selected serotypes of enterobacteriaceae pathogens isolated from polluted ecosystem}

\author{
G. Archana ${ }^{1}$ and Judia Harriet Sumathy $\mathrm{V}^{2}$ \\ ${ }^{1}$ Research Scholar, PG Department of Biotechnology, Women's Christian College, Chennai, India \\ ${ }^{2}$ Associate Professor, PG Department of Biotechnology, Women's Christian College, Chennai, India
}

\begin{abstract}
Salmonella is a genus of rod shaped (bacillus) Gram negative bacteria of the family Enterobacteriaceae. Salmonella species are non-spore-forming, predominantly motile with cell diameters between 0.7 and $1.5 \mu \mathrm{m}$, lengths from 2 to $5 \mu \mathrm{m}$, and peritrichous flagella (all around the cell body). They are chemotrophs, obtaining their energy from oxidation and reduction reactions using organic sources. They are also facultative aerobes, capable of generating ATP with oxygen ("aerobically") when it is available, or when oxygen is not available, using other electron acceptors or fermentation ("anaerobically”). Salmonella species are intracellular pathogens; certain serotypes cause illness. Non-typhoidal serotypes can be transferred from animal-to-human and from human-to-human. They usually invade only the gastrointestinal tract and cause Salmonellosis, the symptoms of which can be resolved without antibiotics. Typhoidal serotypes can only be transferred from human-to-human, and can cause food-borne infection, typhoid fever, and paratyphoid fever. Typhoid fever is caused by Salmonella invading the bloodstream (the typhoidal form), or in addition spreads throughout the body, invades organs, and secretes endotoxins (the septic form). This can lead to life-threatening hypovolemic shock and septic shock, and requires intensive care including antibiotics. The present study is aimed at studying the Serotype of this Enterobacteriaceae pathogen isolated from sewage and drinking water environments.
\end{abstract}

KEY WORDS: ENTEROBACTERIACEAE, SALMONELLA, TYPHOIDAL SEROTYPE, SEWAGE AND DRINKING WATER ENVIRONMENTS

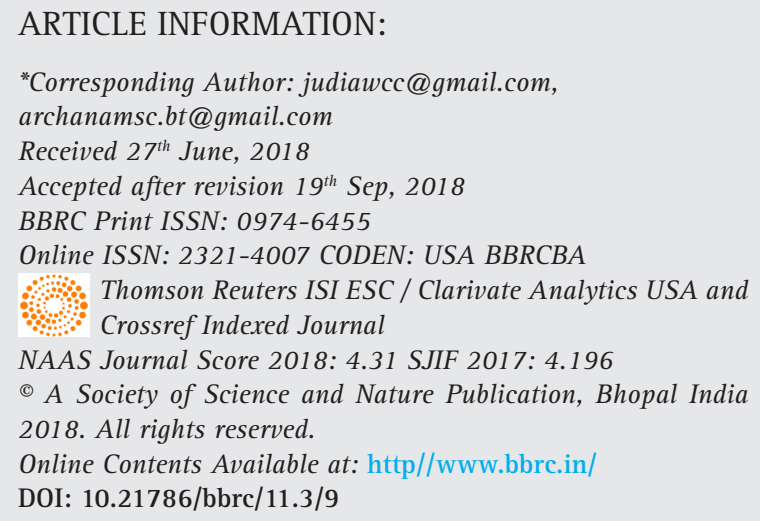




\section{INTRODUCTION}

Salmonella species are facultative intracellular pathogens. A facultative organism uses oxygen to make ATP; when it is not available, it "exercises its option"-the literal meaning of the term-and makes ATP by fermentation, or by substituting one or more of four less efficient electron acceptors as oxygen at the end of the electron transport chain: sulfate, nitrate, sulfur, or fumarate Cabada et al., 1975). Most infections are due to ingestion of food contaminated by animal feces, or by human feces. Salmonella serotypes can be divided into two main groups-typhoidal and non-typhoidal (CDC, 2005).

Non-typhoidal serotypes are more common, and usually cause self-limiting gastrointestinal disease. They can infect a range of animals, and are zoonotic, meaning they can be transferred between humans and other animals (Cooke, and Wain, 2005). Typhoid fever caused by Salmonella serotypes are strictly adapted to humans or higher primates-these include Salmonella Typhi, Paratyphi A, Paratyphi B, and Paratyphi C (D'Aoust, 1989). In the systemic form of the disease, Salmonellae pass through the lymphatic system of the intestine into the blood of the patients (typhoid form) and are carried to various organs (liver, spleen, kidneys) to form secondary foci (septic form). Endotoxins first act on the vascular and nervous apparatus, resulting in increased permeability and decreased tone of the vessels, upset of thermal regulation, and vomiting and diarrhoea (Hudault et al., 2001). In severe forms of the disease, enough liquid and electrolytes are lost to upset the water-salt metabolism, decrease the circulating blood volume and arterial pressure, and cause hypovolemic shock. Septic shock may also develop. Shock of mixed character (with signs of both hypovolemic and septic shock) is more common in severe Salmonellosis. Oliguria and azotemia may develop in severe cases as a result of renal involvement due to hypoxia and toxemia.

Mechanisms of infection differ between typhoidal and nontyphoidal serotypes, owing to their different targets in the body and the different symptoms that they cause. Both groups must enter by crossing the barrier created by the intestinal cell wall, but once they have passed this barrier, they use different strategies to cause infection. Nontyphoidal serotypes preferentially enter M cells on the intestinal wall by bacterial-mediated endocytosis, a process associated with intestinal inflammation and diarrhoea. They are also able to disrupt tight junctions between the cells of the intestinal wall, impairing the cells' ability to stop the flow of ions, water, and immune cells into and out of the intestine. The combination of the inflammation caused by bacterial-medi- ated endocytosis and the disruption of tight junctions is thought to contribute significantly to the induction of diarrhoea (Murray, 1991).

Salmonellae are also able to breach the intestinal barrier via phagocytosis and trafficking by CD18-positive immune cells, which may be a mechanism key to typhoidal Salmonella infection. This is thought to be a more stealthy way of passing the intestinal barrier, and may, therefore, contribute to the fact that lower numbers of typhoidal Salmonella are required for infection than nontyphoidal Salmonella (Olsen et al., 2001). Typhoidal serotypes can use this to achieve dissemination throughout the body via the mononuclear phagocyte system, a network of connective tissue that contains immune cells, and surrounds tissue associated with the immune system throughout the body (Parras et al., 1984).

Salmonellosis is also known to be able to cause back pain or spondylosis. It can manifest as five clinical patterns: gastrointestinal tract infection, enteric fever, bacteremia, local infection, and the chronic reservoir state. The initial symptoms are nonspecific fever, weakness, and myalgia among others. In the bacteremia state, it can spread to any parts of the body and this induces localized infection or it forms abscesses (Popoff, 2001). The forms of localized Salmonella infections are arthritis, urinary tract infection, infection of the central nervous system, bone infection, soft tissue infection, etc.Infection may remain as the latent form for a long time, and when the function of reticular endothelial cells is deteriorated, it may become activated and consequently, it may secondarily induce spreading infection in the bone several months or several years after acute salmonellosis (Silverman, 1979).

\section{MATERIALS AND METHODS}

By plate count method $1 \mathrm{ml}$ of the sample was prepared and transferred to $9 \mathrm{ml}$ of saline and was maintained as master dilution. From this $\left(10^{-1}\right.$ to $\left.10^{-6}\right)$ dilutions were prepared and $1 \mathrm{ml}$ of sample was poured to cool sterilized agar count plate and incubated at $37^{\circ} \mathrm{C}$ for 24 hours. Colony was counted by colony counter. Morphological study was achieved by microscopic observation of Grams staining, Motility test, Catalase test and Oxidase test. A small portion of suspected colony was streaked on medias such as Nutrient Agar, MacConkey Agar and Eosin Methylene Blue Agar. Biochemical tests were performed using Standard Protocol. Following this serological typing was done. Depression plates were taken and were marked as A, B and C. In A depression plate it was marked as negative control in which phenolized saline suspension was added. In B depression 


\begin{tabular}{|c|c|c|}
\hline S. No. & Biochemical Tests & Results \\
\hline 1. & Catalase & Positive \\
\hline 2. & Oxidase & Negative \\
\hline 3. & $\begin{array}{c}\text { TSI Test } \\
\text { Butt } \\
\text { Slant } \\
\text { Gas } \\
\text { H2S }\end{array}$ & $\begin{array}{c}\text { Acid } \\
\text { Acid } \\
\text { Negative } \\
\text { Negative }\end{array}$ \\
\hline 4. & Indole & Negative \\
\hline 5. & Methyl Red & Positive \\
\hline 7. & Voges Proskauer & Positive \\
\hline 8. & Citrate & Positive \\
\hline
\end{tabular}

plate it was marked as test in which phenolized saline suspension and antiserum of respective organism was added and in $\mathrm{C}$ depression plate it was marked as posi- tive control which contain phenolized saline suspension of known organism and antiserum.

\section{RESULTS AND DISCUSSION}

The total number of positive and negative samples obtained from sewage and drinking water for Salmonella paratyphi $A$ was found to be 13 . In identification of bacterial isolate of morphological characteristics by Grams staining and motility for Salmonella paratyphi $A$, it wasfound to be Gram negative small rods and motile. Cultural characteristics of Salmonella paratyphi Aon Nutrient Agar formed large transparent colonies, on Mac Conkey Agar lactose fermenting colonies and on Blood Agar moist colonies. Biochemical Test, Antibiotic Sensitivity Test and Serotype study results indicate the prevalence of Salmonella paratyphi $A$ in the sewage and drinking water samples collected for the present study (Tables $1-4$ and Figures $1-8$ ).

\begin{tabular}{|c|c|c|c|}
\hline S. No & Name of the Antibiotics & Zone of inhibition in $\mathrm{mm}$ & Interpretation \\
\hline 1. & Amikacin ( AI ) & $13 \mathrm{~mm}$ & Resistant \\
\hline 2. & Chloramphenicol ( C ) & $21 \mathrm{~mm}$ & Sensitive \\
\hline 3. & Co - trimoxazole ( CT ) & $13 \mathrm{~mm}$ & Resistant \\
\hline 4. & Tetracycline ( T ) & $20 \mathrm{~mm}$ & Sensitive \\
\hline 5. & Gentamycin ( G ) & $14 \mathrm{~mm}$ & Intermediate \\
\hline 6. & Ceftriaxone ( CTR ) & $22 \mathrm{~mm}$ & Sensitive \\
\hline 7. & Cephotaxime ( CTX ) & No zone & Resistant \\
\hline 8. & Norfloxacin ( NX ) & $18 \mathrm{~mm}$ & Sensitive \\
\hline 9. & Meropenem ( MR ) & No zone & Resistant \\
\hline 10. & Imipenem ( I ) & No zone & Resistant \\
\hline \multicolumn{4}{|c|}{$\begin{array}{l}\text { Zone of inhibition } \\
\text { Below } 10 \mathrm{~mm} \text { - least active } \\
\text { Between } 11-25 \mathrm{~mm} \text { - active } \\
\text { Above } 26 \mathrm{~mm} \text { - very active }\end{array}$} \\
\hline
\end{tabular}

\begin{tabular}{|l|l|l|l|l|l|}
\hline \multirow{2}{*}{ Sable 3. Serotyping of Salmonella paratyphi A from Sewage Sample } \\
\cline { 3 - 7 } & \multirow{2}{*}{ Name of the Isolate } & \multicolumn{5}{|c|}{ Antiserum } \\
\cline { 3 - 7 } & & \multicolumn{3}{|c|}{ Antigen } & H Antigen \\
\cline { 3 - 7 } & & 1 & 2 & 12 & Phase $1-$ a \\
\hline 1. & S. paratyphi $A-1$ & - & + & - & - \\
\hline 2. & S. paratyphi $A-2$ & - & - & + & - \\
\hline 3. & S. paratyphi $A-3$ & - & + & - & - \\
\hline 4. & S. paratyphi $A-4$ & - & - & - & + \\
\hline 5. & S. paratyphi $A-5$ & + & - & - & - \\
\hline 6. & S. paratyphi $A-6$ & - & - & + & - \\
\hline 7. & S. paratyphi $A-7$ & - & - & - & + \\
\hline 8. & S. paratyphi $A-8$ & - & - & - & + \\
\hline
\end{tabular}




\begin{tabular}{|l|l|l|l|l|l|}
\hline \multirow{2}{*}{ Sable 4. Serotypying of Salmonella paratyphi Afrom Drinking Water Sample } \\
\cline { 3 - 6 } & \multirow{2}{*}{ Name of the Isolate } & \multicolumn{5}{|c|}{ Antiserum } \\
\cline { 3 - 6 } & & 1 & 2 & 12 & Phtigen \\
\cline { 3 - 6 } & & - & + & - & - \\
\hline 1. & S. paratyphi $A-1$ & - & - & Antigen \\
\hline 2. & S. paratyphi $A-2$ & + & - & - & - \\
\hline 3. & S. paratyphi $A-3$ & - & - & - & + \\
\hline 4. & S. paratyphi $A-4$ & - & - & + & - \\
\hline 5. & S. paratyphi $A-5$ & - & - & - & + \\
\hline
\end{tabular}
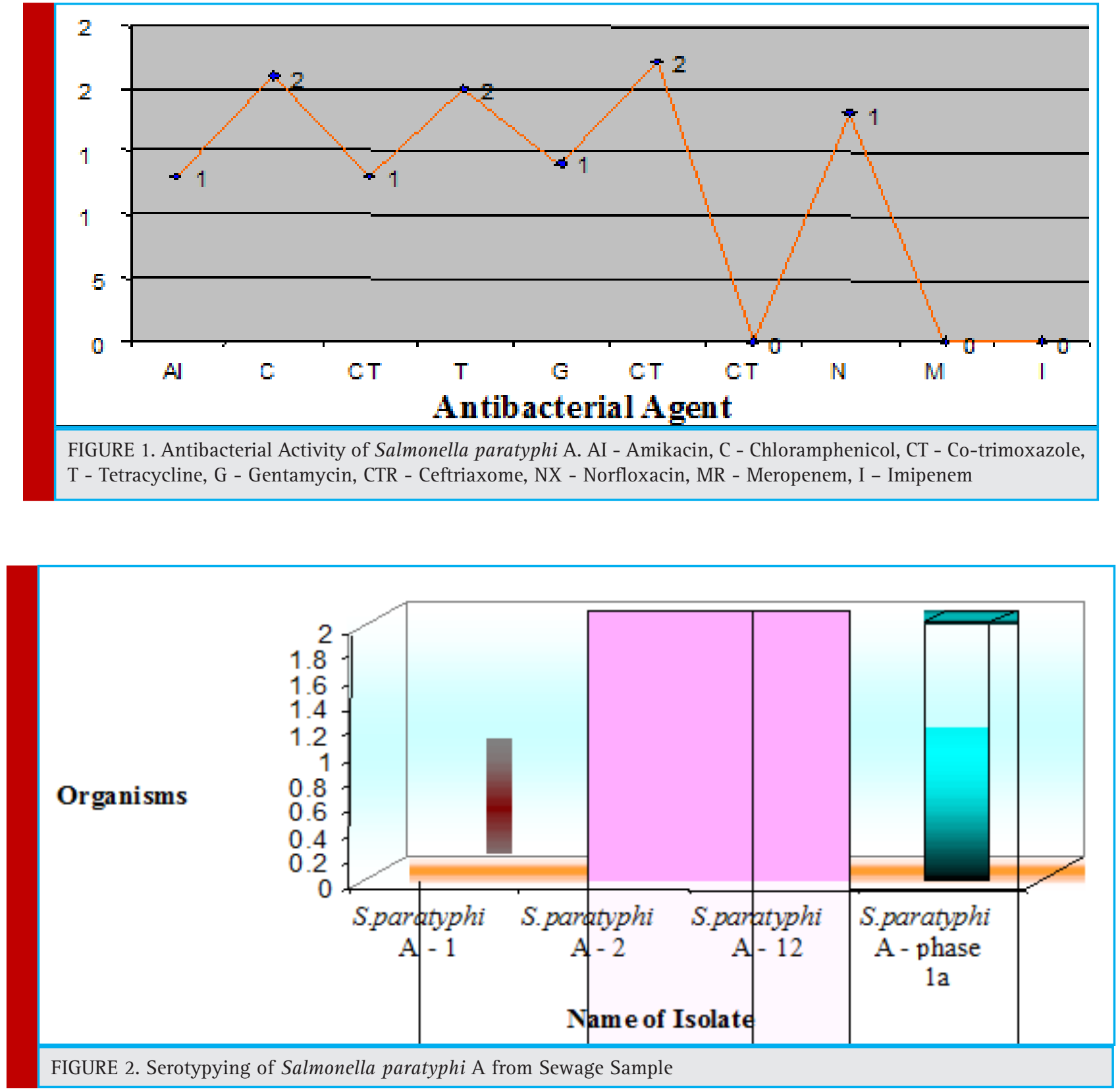


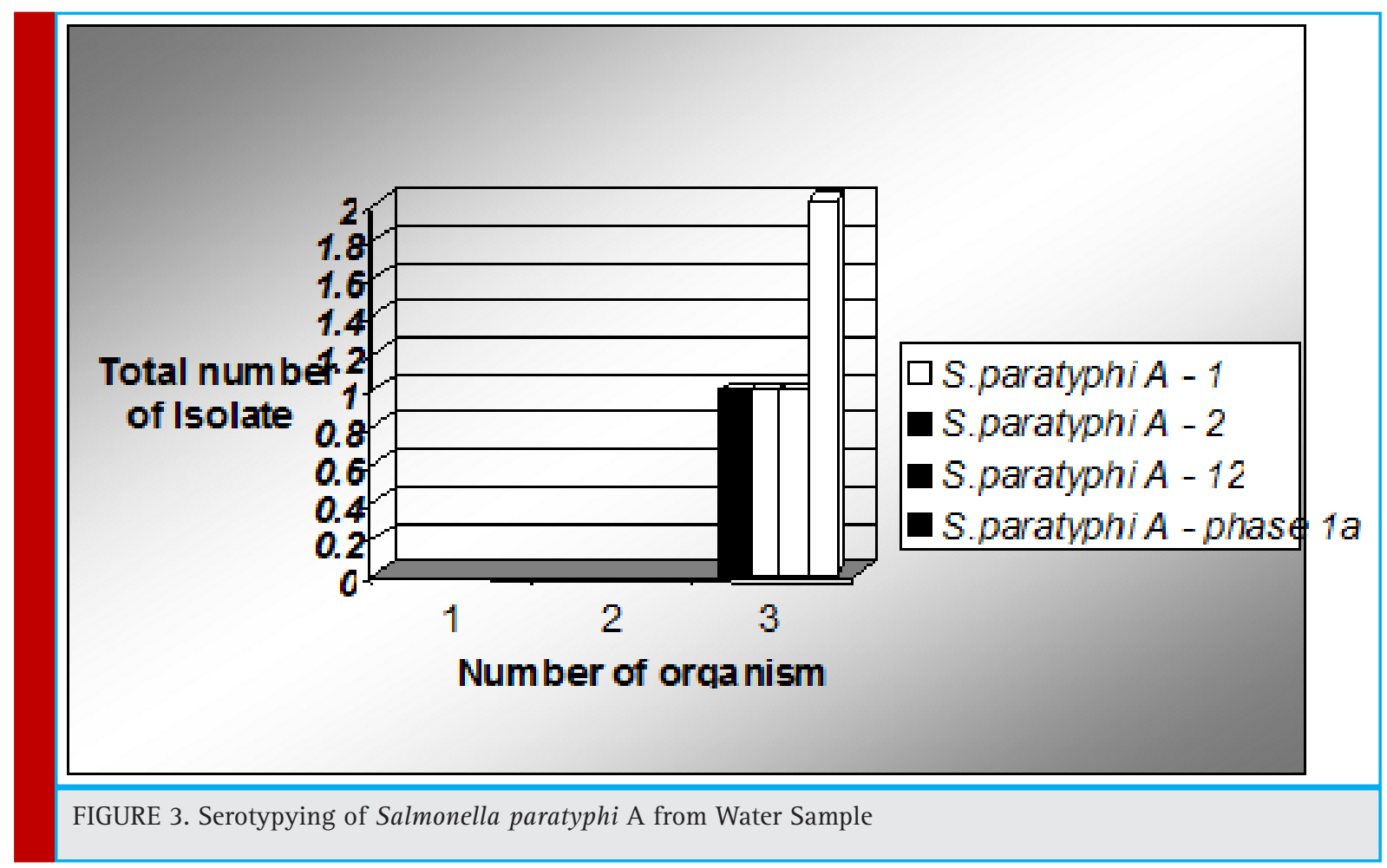

Salmonella infection (salmonellosis) is a common bacterial disease that affects the intestinal tract. Salmonella bacteria typically live in animal and human intestines and are shed through feces. Humans become infected most frequently through contaminated water or food. Typically, people with salmonella infection have no symptoms. Others develop diarrhea, fever and abdominal cramps within eight to 72 hours. Most healthy people recover within a few days without specific treatment. In some cases, the diarrhea associated with salmonella infection can be so dehydrating as to require prompt medical attention. Life-threatening complications also may develop if the infection spreads beyond your intestines. The risk of acquiring salmonella infection is higher if one travels to countries with poor sanitation. Thus the present study was aimed at studying this Enterobacteriacea pathogen which revealed high prevalence pattern in the water samples collected from polluted environments.

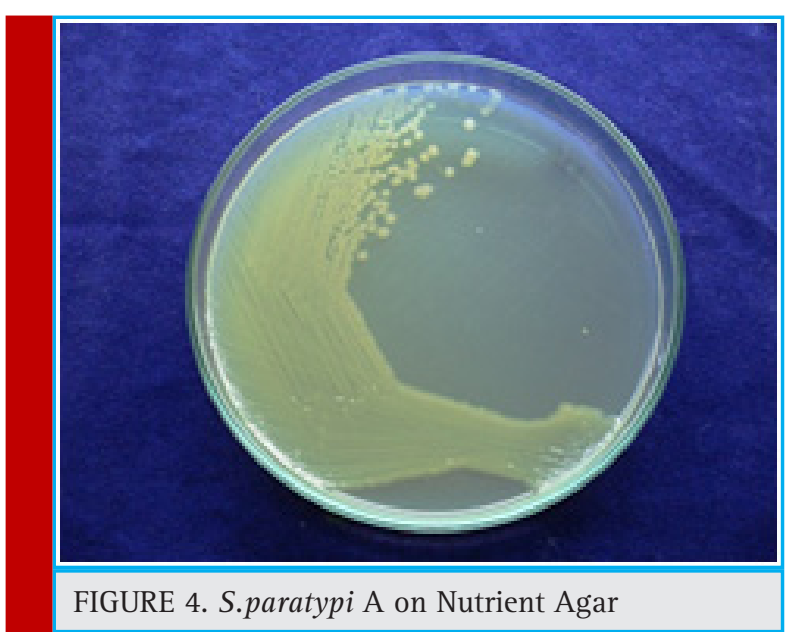

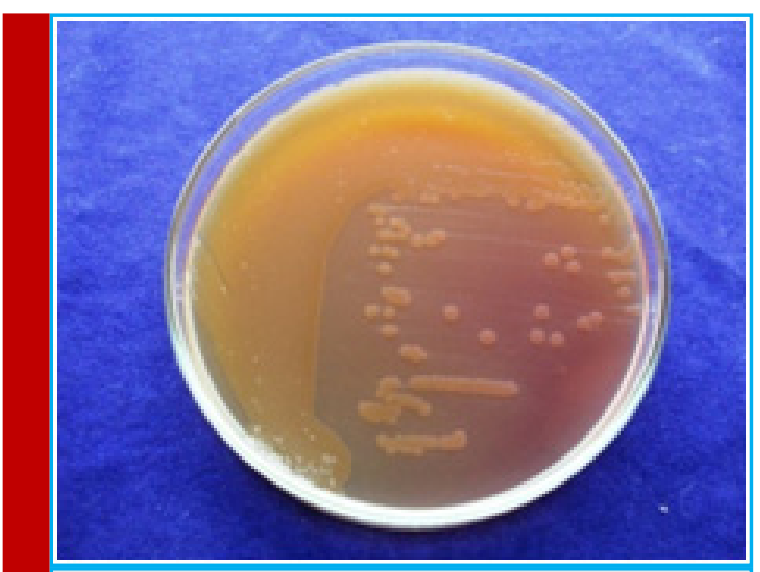

FIGURE 5. S.paratypi A on Nutrient Agar 

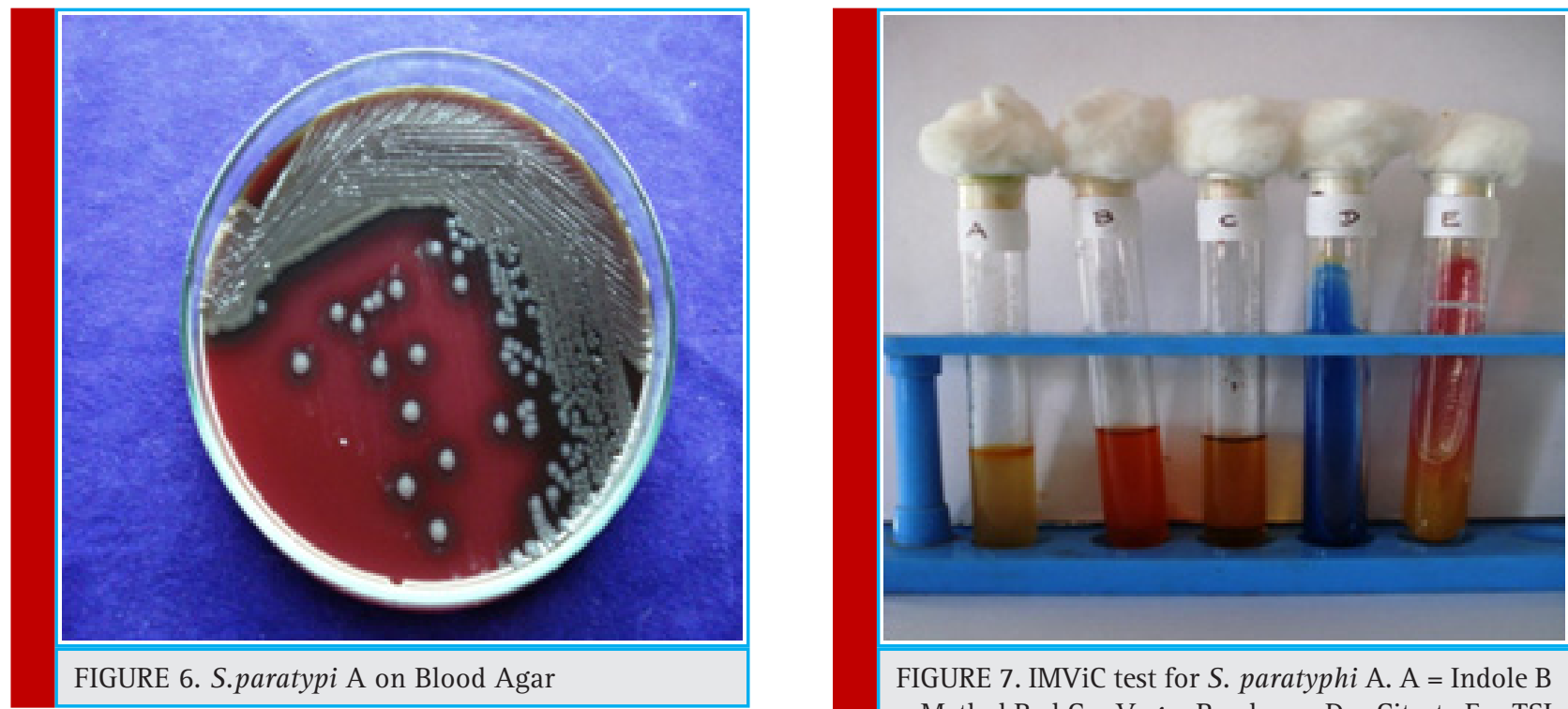

FIGURE 7. IMViC test for $S$. paratyphi $\mathrm{A} . \mathrm{A}=$ Indole $\mathrm{B}$ $=$ Methyl Red C $=$ Voges Proskaur. D = Citrate E $=$ TSI

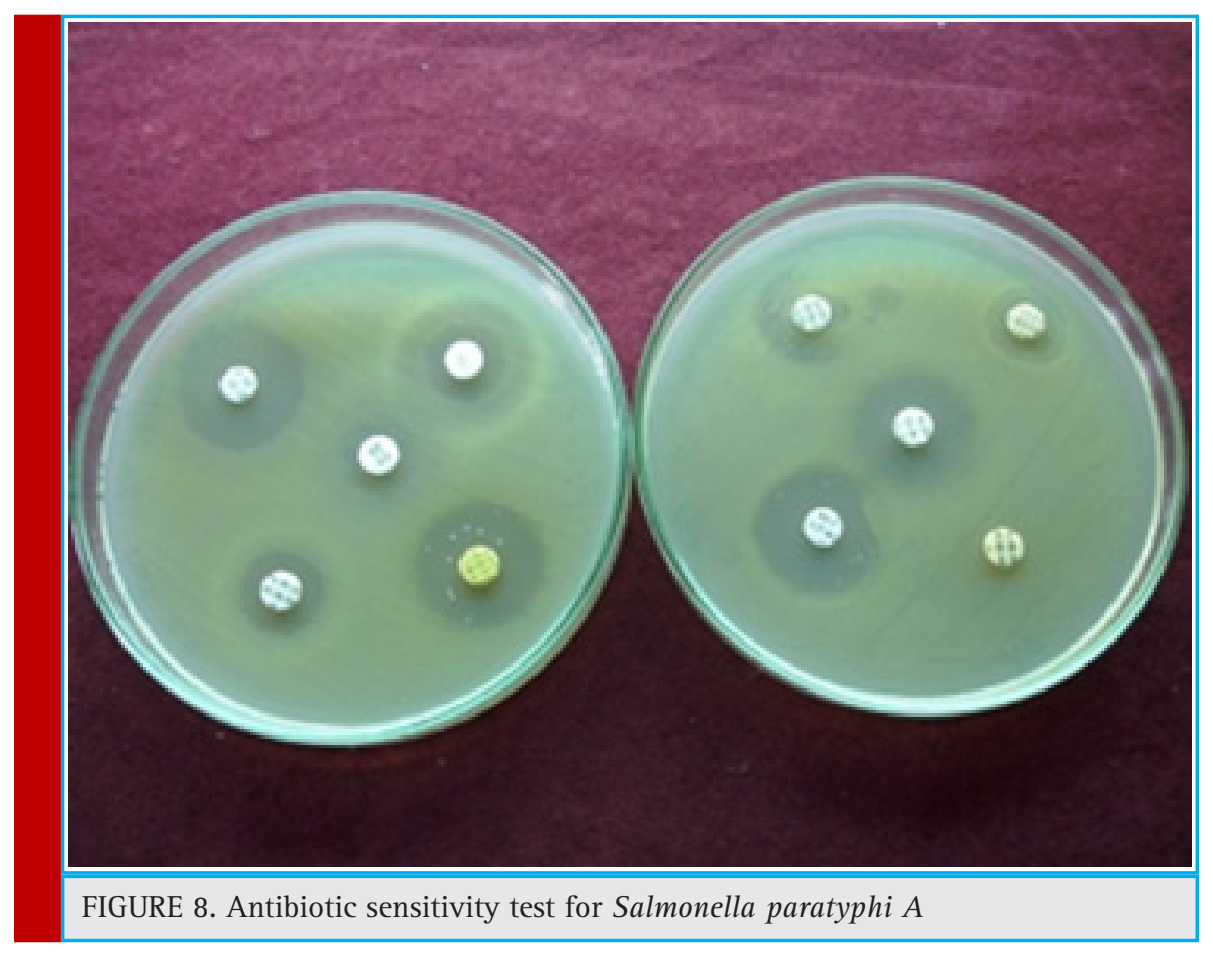

\section{REFERENCES}

Cabadaj, R., Pipova, M. and Turek, P. (1975). Poultry, eggs, and their products as sources of human Salmonellosis in Slovakia. In Proceedings of the World Veterinary Congress. Japan, p. 168.

CDC. (2005). Salmonella Surveillance Annual Summary US Department of Health and Human Services, Centers for Disease Control and Prevention, Atlanta, Georgia.

Cooke, F. and Wain, J. (2005). Antibiotic resistance in Salmonella infections, in Maskell D, Mastroeni P (Eds), Salmonella
Infections. Clinical, immunological and molecular aspects. Cambridge University Press, Cambridge, pp. 25-56.

D’Aoust, J.Y. (1989). Salmonella in Foodborne Bacterial Pathogensed. Doyle, M.P. pp. 327-445. New York, Marcel Dekker.

Hudault, S., Guignot, J. and Servin, A.L. (2001). Escherichia coli strains colonizing the gastrointestinal tract protect germfree mice against Salmonella typhimurium infection. Gut 49:47-55.

Murray, C.J. (1991). Salmonellae in the environment. Revue Scientifique et Technique. Office International des Epizooties 10, 765-785. 
Olsen, S.J., Bishop, R., Brenner, F.W., Roels, T.H., Bean, N. and Tauxe, R.V. (2001). The changing epidemiology of Salmonella trends in serotypes isolated from humans in the United States, 1987-1997. J. Infectious Disease; 183, 753-761.

Parras Jimenez, L., Espigares Garcia, M. and Rodriguez-Contreras, R. (1984). Resistencia de origen plasmidico a los b-lacta micos en el genero Salmonella. Laboratorio 78, 31-41.
Popoff, M.Y. (2001). Antigenic Formulas of the Salmonella Serovars, 8th ed. W.H.O. Collaborating Centre for Reference and Research on Salmonella, Institute Pasteur, Paris, France.

10. Silverman, M. (1979). Phase variation in Salmonella genetic analysis of a recombinational switch. Proc. National Academy Science USA 76, 391-395. 